\title{
Modeling and optimal control for production-regeneration systems - preliminary results -
}

\author{
Farouk Aichouche ${ }^{1,4}$, Nesrine Kalboussi ${ }^{2}$, Alain Rapaport ${ }^{3}$ and Jérôme Harmand ${ }^{1}$
}

\begin{abstract}
This work presents a general model for production - regeneration systems, alternating two functioning modes, the first one as a production process while the second one regenerates the process performances. Such systems are quite common in industry: they include for instance filtration/backwash of membranes, discharge/charge of accumulators. Basically, in these systems the sequence of production/regeneration over time cycles is the manipulating variable. A simple mathematical model involving a single explanatory "hidden variable" to capture the dynamic behavior of the process in both functioning modes is designed. Based on the Pontryagin's Maximum Principle, we characterize the optimal control strategy for a given criterion minimizing the energy consumption under a constraint on the performances.
\end{abstract}

\section{INTRODUCTION}

Many systems in industry require alternating "production" and "regeneration", or "recovering" and "maintenance" modes. It is for example the case of membrane or accumulator systems. During the membrane filtration, the matter is accumulated until a occurring fouling phenomenon. After that, since it is necessary to remove this matter, a regeneration process of the membrane called "backwash phase" is used [1], [2]. Practically, the mass of matter accumulated onto the membrane surface can be measured online, and this variable could be chosen to describe the state of the membrane process in models [3]. In discharge/charge systems, the available energy depends on the quantity of energy stored either electro-chemically, hydraulically (or electro-statically). When the available energy goes down a given threshold, the system has to be recharged. In this case, the variable that best describes the state of the accumulator is the quantity of energy stored (typically a force or an available voltage). In both cases, the state of the system can be described by a single variable that changes monotonously with time: the mass of matter attached onto the membrane or the force (e.g. the voltage) continuously increase during the production phase. Then, during the regeneration or maintenance phase - which also consumes energy and makes the system unavailable for production - the state of the system monotonously decreases (the mass attached onto the membrane or the inverse of the available voltage or force). However, for certain systems

\footnotetext{
${ }^{1}$ F. Aichouche and J. Harmand are with LBE, Univ Montpellier, INRAE, Narbonne, France aichouche.faroukegmail.com, jerome.harmandeinrae.fr

${ }^{2}$ N. Kalboussi is with ITAP, Univ Montpellier, INRAE, Montellier Supagro, France nesrinekalboussiegmail.com

${ }^{3}$ A. Rapaport is with MISTEA, Univ Montpellier, INRAE, France alain.rapaporteinrae.fr

${ }^{4} \mathrm{~F}$. Aichouche is with Laboratory of Automatic Control, Faculty of Technology, University of Tlemcen, Algeria
}

such as electric ones, the two phases could be operated simultaneously, as a convex combination of the dynamics of each phase. We address the control problem which consists in fixing a given performance to be attained (for instance a quantity of fluid to be filtered or a quantity of energy to be used for producing a good), the control variable being the sequence of production/regeneration cycles over time. Notice that there exists necessarily a trade-off between the times spent in production in regeneration phases: the time spent in the production phase brings us closer to our production goal. However, greater the time spent in production lower the efficiency of the process, thus necessitating to switch to the regeneration phase at certain instants. Therefore we shall look for control strategies minimizing the total energy required to attain the target. Such problems have been solved but for maximizing the net water production of a membrane filtration system over a fixed time horizon [5] or the optimal energy management strategies of charge/discharge accumulators [6]. In the present work, the generic modeling of such systems is introduced, and a different cost function where a minimization of energy consumption is considered, once the level of production is fixed. In the literature of production/regeneration systems, the total production with a final constraint - or with free end time was already considered [5], [6]. The paper is organized as follows. First, model and general assumptions are presented leading to an optimal control problem. Then, we apply the Pontryagin's Maximum Principle (PMP), and discuss about singular arc and switching curve for the control synthesis. Finally, a numerical application is presented and discussed before conclusions and perspectives are given.

\section{MODELING AND PRELIMINARIES}

Let $x(t)$ be a variable describing the state of the process at time $t$ - for instance the mass of the membrane deposit during the water filtration or the inverse of available voltage at both terminals of a battery. During the production phase, we can assume that $x(t)$ is growing according to a dynamics $\dot{x}=f_{p}(x)$. During the regeneration phase, this dynamics is reversed according to a dynamics $\dot{x}=-f_{r}(x)$. We consider a control $u(t)$ that takes values 1 during production and -1 during regeneration (or in $[-1,1]$ if a combination of both modes is possible). Then, the controlled dynamics can be written as follows:

$$
\dot{x}=\frac{1+u}{2} f_{p}(x)-\frac{1-u}{2} f_{r}(x), \quad x(0)=x_{0} .
$$


For convenience, we define:

$$
f_{+}(x)=\frac{f_{p}(x)+f_{r}(x)}{2}, \quad f_{-}(x)=\frac{f_{p}(x)-f_{r}(x)}{2}
$$

Equation (1) can be written as:

$$
\dot{x}=f_{-}(x)+u f_{+}(x), \quad x(0)=x_{0} .
$$

Let us assume that the total energy demand is the sum of the energy needed during the production phase and the one required for regeneration: it is given by a function $E_{T}$ that depends on $x(t)$, such that:

$$
E_{T}\left(x_{0}, u(\cdot)\right)=\int_{0}^{t_{f}}\left(\frac{1+u(t)}{2} l_{p}(x(t))+\frac{1-u(t)}{2} l_{r}(x(t)) d t\right.
$$

where $l_{p}$ and $l_{r}$ are the energies per unit of time required respectively for the production and the the regeneration.

It is further assumed that the energy needed for production or regeneration is proportional to a flux (more generally a quantity of something over a period of time) over an entity playing the role of a "resistance". The functions $l_{p}$ and $l_{r}$ can then be expressed as:

$$
l_{p}(x)=Q_{p} \tau_{p}(x), \quad l_{r}(x)=Q_{R} \tau_{r}(x)
$$

where $Q_{p}$ and $Q_{r}$ are typically (constant) fluxes while the functions describing the way that the state affects production and regeneration phases are $\tau_{p}(\cdot)$ and $\tau_{r}(\cdot)$, respectively. One usually considers that the regeneration speed is higher than the wear or fouling speed. This amounts to require the following assumption.

Assumption 2.1: One has $f_{r}^{\prime}(x)>f_{p}^{\prime}(x)$ for any $x>0$.

For convenience, we define:

$$
l_{+}(x)=\frac{l_{p}(x)+l_{r}(x)}{2}, \quad l_{-}(x)=\frac{l_{p}(x)-l_{r}(x)}{2}
$$

Then, equation (3) can be simply written as:

$$
E_{T}\left(x_{0}, u(\cdot)\right)=\int_{0}^{t_{f}}\left(l_{+}(x(t))+u(t) l_{-}(x(t))\right) d t
$$

Given an initial state $x_{0} \geq 0$, the objective is to determine an optimal strategy $u(\cdot)$ that takes values -1 or 1 for minimizing $E_{T}\left(x_{0}, u(\cdot)\right)$ for a given total production $p^{*}$.

The process stops at the first time $t_{f}$ for which the target is reached: $p\left(t_{f}\right)=p^{*}$, where the production $p(t)$ at time $t$ is given by

$$
p(t)=\int_{0}^{t}\left(\frac{1+u(t)}{2} Q_{p}-\frac{1-u(t)}{2} Q_{r}\right) d t
$$

The production dynamics is then

$$
\dot{p}=\frac{1+u}{2} Q_{p}-\frac{1-u}{2} Q_{r}, \quad p(0)<p^{\star}
$$

For convenience, we define:

$$
Q_{+}=\frac{Q_{p}+Q_{r}}{2}, \quad Q_{-}=\frac{Q_{p}-Q_{r}}{2}
$$

Equation (7) can be written as:

$$
\dot{p}=Q_{-}+u Q_{+}, \quad p(0)<p^{\star}
$$

To summarize, we consider the following optimal control problem:

$$
\begin{aligned}
& \inf _{u(\cdot)} \int_{0}^{t_{f}}\left(l_{+}(x(t))+u(t) l_{-}(x(t))\right) d t \text { subject to } \\
& \left\{\begin{array}{l}
\dot{x}=f_{-}(x)+u f_{+}(x), \quad x(0)=x_{0} \\
\dot{p}=Q_{-}+u Q_{+}, \quad p(0)=p_{0}
\end{array} \quad u \in[-1,1]\right.
\end{aligned}
$$

where $t_{f}$ is the first entry time in the target

$$
\mathscr{T}=\left\{(x, p) \text { s.t. } p \geq p^{\star}\right\}
$$

Remark 2.1: As one has $Q_{+}>0$ and $Q_{+}>Q_{-}$, the target can be reached in finite time, taking for instance the control $u=1$ at any time. However, it is well known from the theory of optimal control that the existence of an optimal trajectory can be guaranteed when the velocity set is convex. Therefore, we shall consider for the mathematical analysis that the control $u(\cdot)$ can take values in the interval $[-1,1]$. The question of practical applicability of a control that takes values different to -1 and 1 relies then on bang-bang approximations.

When a membrane operates in filtration, the flow resistance is never null and increases according to the mass $x$ formed on the membrane surface, which subsequently decreases the permeate flux. Thus, we assume that the rate $f_{p}$ at which the mass of material adheres to the membrane surface during filtration is a positive decreasing function. When changing to backwash operation, the mass is decomposed and the membrane's permeability increases again. Therefore, the mass detachment can be described by a positive increasing function $f_{r}$.

For simplicity, we consider in this study the simple expressions of the functions $f_{p}, f_{r}, l_{p}, l_{r}$ :

$$
\begin{aligned}
& f_{p}(x)=-a_{p} x+b_{p}, \quad f_{r}(x)=a_{r} x \\
& l_{p}(x)=c_{p} x+d_{p}, \quad l_{r}=c_{r} x+d_{r}
\end{aligned}
$$

where $a_{p}, b_{p}, a_{r}, c_{p}, d_{p}, c_{r}, d_{r}$ are non negative parameters such that the functions $f_{p}, f_{r}, l_{p}, l_{r}$ are not identically null. One can straightforwardly check that the domain $\{(x, p)$ s.t. $x>0\}$ is invariant by the dynamics (2) whatever is the control law $u(\cdot)$. Assumption 2.1 is fulfilled when one has

$$
a_{r}>a_{p}
$$

Notice that the interval $\left(0, x_{\max }\right)$ with

$$
x_{\text {max }}=\frac{b_{p}}{a_{p}} \quad\left(+\infty \text { if } a_{p}=0\right)
$$

is invariant by the dynamics (1) and that $p(\cdot)$ is increasing whatever is the control. Therefore the domain

$$
\mathscr{D}=\left[0, x_{\max }\right] \times\left[0, p^{\star}\right)
$$

is invariant and we shall consider initial conditions $\left(x_{0}, p_{0}\right)$ in the domain $\mathscr{D}$ only. 


\section{Application OF THE MAXIMUM PRINCIPLE}

Let us first introduce the Hamiltonian of the system:

$$
\begin{aligned}
& H\left(x, \lambda_{x}, \lambda_{p}, u\right)=\lambda_{x}\left[f_{-}(x)+u f_{+}(x)\right] \\
& +\lambda_{p}\left[Q_{-}+u Q_{+}\right]+\lambda_{0}\left[l_{+}(x)+u l_{-}(x)\right]
\end{aligned}
$$

where $\lambda_{0}$ is equal to -1 or 0 . The PMP states that for any optimal solution $u^{\star}(\cdot)$ there exists adjoint variables $\lambda_{x}(\cdot)$, $\lambda_{p}(\cdot)$ solutions of the adjoint system

$$
\left\{\begin{array}{l}
\dot{\lambda}_{x}(t)=-\partial_{x} H\left(x(t), \lambda_{x}(t), \lambda_{p}(t), u^{\star}(t)\right) \\
\dot{\lambda}_{p}(t)=-\partial_{p} H\left(x(t), \lambda_{x}(t), \lambda_{p}(t), u^{\star}(t)\right)
\end{array}\right.
$$

where $u^{\star}(t)$ maximizes $u \mapsto H\left(x(t), \lambda_{x}(t), \lambda_{p}(t), u\right)$ at almost any $t \in\left[0, t_{f}\right]$, with the transversality conditions

$$
\left(\begin{array}{l}
\lambda_{x}\left(t_{f}\right) \\
\lambda_{p}\left(t_{f}\right)
\end{array}\right) \in-N_{\mathscr{T}}\left(x\left(t_{f}\right), p\left(t_{f}\right)\right)
$$

(where $N_{\mathscr{T}}$ denotes the normal cone to the set $\mathscr{T}$ ). Moreover, the vector $\left(\lambda_{x}(\cdot), \lambda_{p}(\cdot), \lambda_{0}\right)$ is not identically null and one has

$$
\bar{H}(t)=\max _{u \in[-1,1]} H\left(x(t), \lambda_{x}(t), \lambda_{p}(t), u\right)=0, t \in\left[0, t_{f}\right]
$$

(see for instance [4]). Here, the adjoint equations are

$\dot{\lambda}_{x}=-\lambda_{x} f_{-}^{\prime}(x)-\lambda_{0} l_{+}^{\prime}(x)-u^{\star}\left(\lambda_{x} f_{+}^{\prime}(x)+\lambda_{0} l_{-}^{\prime}(x)\right)$

$\dot{\lambda}_{p}=0$

with the transversality conditions

$$
\lambda_{x}\left(t_{f}\right)=0, \quad \lambda_{p}\left(t_{f}\right) \geq 0
$$

From (11), we define the switching function

$$
\phi(t)=\lambda_{x}(t) f_{+}(x(t))+\lambda_{p} Q_{+}+\lambda_{0} l_{-}(x(t))
$$

which gives the following maximization condition

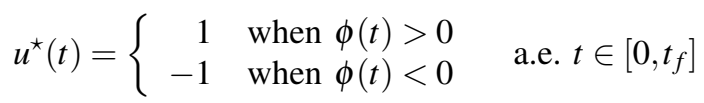

We begin by a Lemma about $\lambda_{0}$ and $\lambda_{p}$.

Lemma 3.1: For any optimal solution, one has the following properties

i. $\lambda_{0}=-1$ i.e. there does not exist abnormal extremal,

ii. there exists $\bar{t}<t_{f}$ such that $u^{\star}(t)=1$ is optimal for $t \in\left[\bar{t}, t_{f}\right]$,

iii. $\lambda_{p}$ is a positive constant.

Proof: i. If $\lambda_{0}=0$, the solution $\lambda_{x}(\cdot)$ of (13) for the terminal condition (15) is identically equal to zero. Then, $\lambda_{p}$ is non null and the Hamiltonian is constant equal to $\lambda_{p}\left(Q_{-}+\right.$ $Q_{+}$) which contradicts (12).

ii. At terminal time, the terminal conditions (15) give $\phi\left(t_{f}\right)=\lambda_{p} Q_{+}-l_{-}\left(x\left(t_{f}\right)\right)$, where $\lambda_{p}$ is non negative and $l_{-}\left(x\left(t_{f}\right)\right.$ is negative under Assumption 2.1. Therefore $\phi\left(t_{f}\right)>$ 0 and by continuity of $\phi(\cdot)$, we conclude from (17) that there exists $\bar{t}<t_{f}$ such that $u^{\star}(t)=1$ is optimal for $t \in\left[\bar{t}, t_{f}\right]$.

iii. If $\lambda_{p}=0$, consider the optimal control $u^{\star}(t)=1$ for $t \in\left[\bar{t}, t_{f}\right]$. On this time interval one has then

$$
\begin{aligned}
& H\left(x(t), \lambda_{x}(t), \lambda_{p}, u^{\star}(t)\right)= \\
& \quad \lambda_{x}(t)\left(f_{-}(x(t))+f_{+}(x(t))\right)-\left(l_{+}(x(t))+l_{-}(x(t))\right)
\end{aligned}
$$

which is a continuous function of $t$. The terminal conditions (15) give then $H\left(t_{f}\right)=-\left(l_{+}\left(x\left(t_{f}\right)\right)+l_{-}\left(x\left(t_{f}\right)\right)\right)<0$. Therefore condition (12) is violated on $\left[\bar{t}, t_{f}\right]$.

We focus now on the study of singular arcs, locus for which $\phi$ is null on a time interval of non null measure.

\section{Singular ARCS}

For simplicity we drop the time dependency:

$$
\begin{gathered}
\dot{x}=\frac{-\left(a_{p}+a_{r}\right) x+b_{p}}{2}+u \frac{\left(-a_{p}+a_{r}\right) x+b_{p}}{2} \\
\dot{\lambda}_{x}=\lambda_{x} \frac{a_{p}+a_{r}}{2}+\frac{c_{p}+c_{r}}{2}+u\left(\frac{a_{p}-a_{r}}{2} \lambda_{x}+\frac{c_{p}-c_{r}}{2}\right) \\
\phi=\lambda_{x} \frac{\left(a_{r}-a_{p}\right) x+b_{p}}{2}+\lambda_{p} Q_{+}+\frac{\left(c_{r}-c_{p}\right) x-d_{p}+d_{r}}{2} \\
H=\lambda_{x} \frac{-\left(a_{P}+a_{r}\right) x+b_{p}}{2}+\lambda_{p} Q_{-}-\frac{\left(c_{p}+c_{r}\right) x+d_{p}+d_{r}}{2}+u \phi
\end{gathered}
$$

Conditions $\phi=0$ and $H=0$ give together the system of two equations in $\left(\lambda_{x}, \lambda_{v}\right)$ :

$$
\left\{\begin{array}{l}
\left(\left(a_{r}-a_{p}\right) x+b_{p}\right) \lambda_{x}+2 Q_{+} \lambda_{p}=\left(c_{p}-c_{r}\right) x+d_{p}-d_{r} \\
\left(-\left(a_{P}+a_{r}\right) x+b_{p}\right) \lambda_{x}+2 Q_{-} \lambda_{p}=\left(c_{p}+c_{r}\right) x+d_{p}+d_{r}
\end{array}\right.
$$

Its discriminant $\Delta(x)$ is given by the following expression

$$
\Delta(x)=\left(a_{p} Q_{r}+a_{r} Q_{p}\right) x-b_{p} Q_{r}
$$

which is null for the particular value

$$
\bar{x}_{\Delta}=\frac{b_{p} Q_{r}}{a_{p} Q_{r}+a_{r} Q_{p}}
$$

If $\phi$ is null when $x \neq \bar{x}_{\Delta}$, the system (18) admits an unique solution, for which a direct computation gives

$$
\bar{\lambda}_{x}(x)=\frac{P_{x}(x)}{\Delta(x)}, \quad \bar{\lambda}_{p}(x)=\frac{P_{p}(x)}{\Delta(x)}
$$

with

$$
\left\{\begin{array}{l}
P_{x}(x)=-\left(c_{p} Q_{r}+c_{r} Q_{p}\right) x-\left(d_{p} Q_{r}+d_{r} Q_{p}\right) \\
P_{p}(x)=\left(a_{r} c_{p}-a_{p} c_{r}\right) x^{2}+\left(a_{r} d_{p}-a_{p} d_{r}+b_{p} c_{r}\right) x+b_{p} d_{r}
\end{array}\right.
$$

As $\lambda_{p}$ is constant along any optimal trajectory, we deduce that $\phi$ can be null only at isolated values $\bar{x}$, positive roots of the polynomial

$$
P_{p}(x)-\lambda_{p} \Delta(x)=0
$$

In any case, a singular arc is possible only for constant values of $x$ equal to $\bar{x}_{\Delta}$ or $\bar{x}$ with $\Delta(\bar{x}) \neq 0$. Then, the corresponding value of the control for the trajectory to stay at $x=\bar{x}$ is

$$
\bar{u}=-\frac{f_{-}(\bar{x})}{f_{+}(\bar{x})}=\frac{-\left(a_{p}+a_{r}\right) \bar{x}+b_{p}}{\left(a_{r}-a_{p}\right) \bar{x}+b_{p}}
$$

A straightforward computation gives

$$
\dot{\phi}=\frac{1}{2}\left(a_{r} b_{p} \lambda_{x}+\left(a_{r} c_{p}-a_{p} c_{r}\right) x+b_{p} c_{r}\right)
$$

that we write using the expression of $\phi$ as follows:

$$
\dot{\phi}=\frac{a_{r} b_{p}}{\left(a_{r}-a_{p}\right) x+b_{p}} \phi+\psi\left(x, \lambda_{p}\right)
$$


where the function $\psi$ has the expression

$$
\begin{array}{r}
\psi\left(x, \lambda_{p}\right)=\frac{1}{2} \frac{M\left(x, \lambda_{p}\right)}{\left(a_{r}-a_{p}\right) x+b_{p}} \text { with } \\
M\left(x, \lambda_{p}\right)=\left(a_{p}-a_{r}\right)\left(a_{p} c_{r}-a_{r} c_{p}\right) x^{2}-2\left(a_{p} c_{r}-a_{r} c_{p}\right) b_{p} x \\
\quad+a_{r} b_{p}\left(d_{p}-d_{r}-2 \lambda_{p} Q_{+}\right)+b_{p}^{2} c_{r}
\end{array}
$$

Notice from condition (10) that the denominator in expression (22) is always positive and thus $\psi$ is well defined. Therefore a singular arc $x=\bar{x}$ with $x \neq \bar{x}_{\Delta}$ has to satisfy

$$
\Gamma(\bar{x})=\psi\left(\bar{x}, \bar{\lambda}_{p}(\bar{x})\right)=0
$$

Finally, a straightforward but lengthy computation (verified with Maple) gives the following expression of $\Gamma$

$$
\begin{gathered}
\Gamma(x)=-\frac{1}{2} \frac{N(x)}{\Delta(x)} \text { with } \\
N(x)=-\left(a_{r} c_{p}-a_{p} c_{r}\right)\left[\left(a_{p} Q_{r}+a_{r} Q_{p}\right) x^{2}-2 b_{p} Q_{r} x\right] \\
+a_{r} b_{p}\left(d_{p} Q_{r}+d_{r} Q_{p}\right)+b_{p}^{2} c_{r} Q_{r}
\end{gathered}
$$

Consequently, a singular arc $x=\bar{x}$ with $\bar{x} \neq \bar{x}_{\Delta}$ is a root of $N$. We consider that the case for which $\bar{x}_{\Delta}$ could be also a root of $N$ is non generic. Notice that the signs of the coefficients of the polynomial $N$ ensures that when real roots exists, at least one is positive. Moreover, when the condition

$$
a_{r} c_{p}-a_{p} c_{r}>0
$$

is fulfilled, there is exactly one positive root given by

$$
\bar{x}=\frac{b_{p} Q_{r}+\sqrt{b_{p}^{2} Q_{r}^{2}+\frac{a_{r} b_{p}\left(d_{p} Q_{r}+d_{r} Q_{p}\right)+b_{p}^{2} c_{r} Q_{r}}{a_{r} c_{p}-a_{p} c_{r}}}}{a_{p} Q_{r}+a_{r} Q_{p}}
$$

However, accordingly to Lemma 3.1.iii, an arc $x=\bar{x}$ can be part of an optimal solution only if its corresponding adjoint $\bar{\lambda}_{p}(\bar{x})$, given by expression (19), is positive.

Consider for any root $\bar{x}$ of $N$ with $\lambda_{p}(\bar{x})>0$ the function

$$
\gamma_{\bar{x}}(x)=\psi\left(x, \lambda_{p}(\bar{x})\right)
$$

Let us show that if it is non increasing in the neighborhood of $\bar{x}$, then the arc $x=\bar{x}$ cannot belong to an optimal solution. Indeed, if $x=\bar{x}$ is reached from below at a time $t_{s}<t_{f}$, then $u(t)=1$ is optimal on any time interval $\left(t_{s}-\delta, t_{c}\right)$ with $\delta$ sufficiently enough, and therefore $\phi$ has to be positive for $\delta$ small enough (as the locus where $\phi$ can change its sign are isolated). An another hand, $\gamma_{\bar{x}}$ non increasing implies that for $\delta$ small enough $\gamma_{\bar{x}}$ is non negative on $\left(t_{s}-\delta, t_{s}\right)$. This implies from (21) that $\phi$ cannot decrease on the interval $\left(t_{s}-\delta, t_{s}\right)$ and therefore cannot change its sign at $\bar{t}$. A similar argumentation applies if $x=\bar{x}$ is reached from above.

Consider now the candidate singular arc $x=\bar{x}_{\Delta}$. Substituting this value in (21) for $\dot{\phi}=0$ gives the corresponding value of $\lambda_{x}$, and then $\phi=0$ provides the value of $\lambda_{p}$ :

$$
\begin{aligned}
& \bar{\lambda}_{x}\left(x_{\Delta}\right)=-\frac{c_{p} Q_{r}+c_{r} Q_{p}}{a_{p} Q_{r}+a_{r} Q_{p}} \\
& \bar{\lambda}_{p}\left(x_{\Delta}\right)=-\frac{C}{\left(a_{p} Q_{r}+a_{r} Q_{p}\right)^{2}\left(Q_{p}+Q_{r}\right)}
\end{aligned}
$$

$$
\text { with } \begin{aligned}
C & =a_{r}\left(a_{r}\left(d_{r}-d_{p}\right)-b_{p} c_{r}\right) Q_{p}^{2} \\
& +2 a_{r}\left(a_{p}\left(d_{r}-d_{p}\right)-b_{p} c_{p}\right) Q_{p} Q_{r} \\
& +\left(a_{p}^{2}\left(d_{r}-d_{p}\right)+\left(a_{p} c_{r}-c_{p}\left(a_{p}+a_{r}\right)\right) b_{p}\right) Q_{r}^{2}
\end{aligned}
$$

Therefore, if the number $C$ is positive, the arc $x=x_{\Delta}$ cannot be part of an optimal solution.

Notice that generically, roots of $N$ and $x_{\Delta}$ have different values of $\bar{\lambda}_{p}(\bar{x})$. Therefore, an optimal solution cannot have more than one singular arc. Notice also that as $u(t)=1$ is optimal on the interval $\left[\bar{t}, t_{f}\right]$ (cf Lemma 3.1.iii) and $\bar{H}$ is null at any time, this imposes the final state $\bar{x}_{f}=x\left(t_{f}\right)$ to satisfy the condition $\lambda_{p} Q_{p}-l_{p}\left(\bar{x}_{f}\right)=0$, that is

$$
\bar{x}_{f}=\frac{\lambda_{p}(\bar{x}) Q_{p}-d_{p}}{c_{p}}
$$

Finally, if an optimal solution possesses a singular arc, this imposes that the singular arc is left with $p=\bar{p}$ such that

$$
\begin{aligned}
\bar{p} & =p^{\star}-Q_{p} \int_{\bar{x}}^{\bar{x}_{f}} \frac{d x}{f_{p}(x)} \\
& =p^{\star}-\left\{\begin{array}{l}
\frac{Q_{p}}{a_{p}} \log \left(\frac{-a_{p} \bar{x}+b_{p}}{-a_{p} \bar{x}_{f}+b_{p}}\right) \text { if } a_{p}>0 \\
\frac{Q_{p}}{b_{p}}\left(\bar{x}_{f}-\bar{x}\right) \text { if } a_{p}=0
\end{array}\right.
\end{aligned}
$$

\section{SWITCHING CURVE}

We know from Lemma 3.1 that for any optimal solution, the control $u(t)=1$ is optimal on an interval $\left[\bar{t}, t_{f}\right]$. Therefore, for each possible terminal state $x_{f}$ in $\left[0, x_{\text {max }}\right]$, one can determine in backward time the possible locus in the $(p, x)$ plane where the switching function $\phi$ changes its sign (if this happens). This defines a curve $\mathscr{C}$ (when it exists), where an optimal control switches from -1 to 1 or from $\bar{u}$ to 1 (when $x=\bar{x}$ is a singular arc). We characterize this curve.

For the control $u=1$, one has

$$
\begin{gathered}
\dot{x}=-a_{p} x+b, \quad \dot{p}=Q_{p} \quad \text { and } \\
H=\lambda_{x}\left(-a_{p} x+b\right)+\lambda_{p} Q_{p}-c_{p} x-d_{p}
\end{gathered}
$$

For $x_{f}=x\left(t_{f}\right)$ one can determine from $H=0$ and the terminal condition $\lambda_{x}\left(t_{f}\right)=0$ the value of the constant $\lambda_{p}$ as a function of $x_{f}$ :

$$
\lambda_{p}^{\dagger}\left(x_{f}\right)=\frac{c_{p} x_{f}+d_{p}}{Q_{p}}
$$

From $H=0$, the adjoint $\lambda_{x}$ can be determined as a function of $x_{f}$ and $x$ (as long as the switching function is positive):

$$
\lambda_{x}^{\dagger}\left(x_{f}, x\right)=-\frac{c_{p}\left(x_{f}-x\right)}{b_{p}-a_{p} x}
$$

Then, the switching function $\phi$ can be also expressed as a function of $x_{f}$ and $x$ :

$$
\begin{aligned}
& \phi^{\dagger}\left(x_{f}, x\right)=\frac{1}{2 Q_{p}\left(a_{p} x-b_{p}\right)}\left(a_{p} c_{r}-a_{r} c_{p}\right) Q_{p} x^{2} \\
& \quad+\left(c_{p}\left(a_{p} Q_{r}+a_{r} Q_{p}\right) x_{f}+a_{p}\left(d_{r} Q_{p}+d_{p} Q_{r}\right)-b_{p} c_{r} Q_{p}\right) x \\
& \quad-b_{p}\left(c_{p} Q_{r} x_{f}+d_{p} Q_{r}+d_{r} Q_{p}\right)
\end{aligned}
$$


Consider then the root $x_{c}$ of the polynomial $x \mapsto \phi\left(x_{f}, x\right)$ which is the closest to $x_{f}$ (if it exists). It corresponds to the value of $x$ where the function $\phi$ changes it sign for the first backward time. The corresponding value of $p$ is then

$$
p_{c}=p^{\star}-\left\{\begin{array}{l}
\frac{Q_{p}}{a_{p}} \log \left(\frac{-a_{p} x_{c}+b_{p}}{-a_{p} x_{f}+b_{p}}\right) \text { if } a_{p}>0 \\
\frac{Q_{p}}{b_{p}}\left(x_{f}-x_{c}\right) \text { if } a_{p}=0
\end{array}\right.
$$

and $\left(p_{c}, x_{c}\right)$ belongs then to $\mathscr{C}$. Therefore, the curve $\mathscr{C}$ (if it exists) can be parameterized by the final value $x_{f}$.

Lemma 5.1: The curve $\mathscr{C}$ (when it exists) is such that

1) $x_{f} \mapsto x_{c}$ is increasing,

2) $\lim _{x_{f} \rightarrow x_{\max }} x_{c}\left(t_{f}\right)=x_{\max }$

Proof: 1) If $x_{f} \mapsto x_{c}$ is not increasing, there exists $x_{f_{2}}>x_{f_{1}}$ such that $x_{c}\left(x_{f_{2}}\right) \leq x_{c}\left(x_{f_{1}}\right)$. As the trajectories of the dynamics with $u=+1$ cannot cross in the $(p, x)$ plane, one has necessarily $p_{c}\left(x_{f_{2}}\right)<p_{c}\left(x_{f_{1}}\right)$. Then, the trajectory with $u=-1$ reaching the switching point $\left(p_{c}\left(x_{f_{1}}\right), x_{c}\left(x_{f_{1}}\right)\right)$ has to cross the trajectory joining $\left(p_{c}\left(x_{f_{1}}\right), x_{c}\left(x_{f_{1}}\right)\right)$ to $\left(p^{\star}, x_{f 2}\right)$ with $u=1$, that is in contradiction with the definition of $\mathscr{C}$. 2) As $x_{f} \mapsto x_{c}$ is increasing, there exists a limit $\bar{x}_{c}$ of $x_{c}$ when $x_{f}$ tends to $x_{\max }$ (which is less or equal to $x_{\max }$ ). Let $\bar{p}_{c}$ be the corresponding value of $p_{c}$. If $\bar{x}_{c}<x_{\max }$, consider then the trajectory in the $(p, x)$ plane that arrives to $\left(\bar{p}_{c}, \bar{x}_{c}\right)$ with the control $u=-1$ and leave $\left(\bar{p}_{c}, \bar{x}_{c}\right)$ with $u=1$. As one has $\dot{x}<0$ wit $u=-1$ and $\dot{x}>0$ with $u=1$, this trajectory belongs to the subset $\left\{(p, x), x \geq \bar{x}_{c}\right\}$. At any point $(p, x)$ which is above this trajectory, the control $u=-1$ cannot be optimal because then it has to switch to $u=1$ outside $\mathscr{C}$. Therefore, the trajectory that arrives to $\left(\bar{p}_{c}, \bar{x}_{c}\right)$ with the control $u=-1$ is a locus of commutation with $x>\bar{x}_{c}$, which contradicts $\bar{x}_{c}<x_{\max }$.

When a singular arc $x=\bar{x}$ is optimal, $(\bar{p}, \bar{x})$ necessarily belongs to $\mathscr{C}$. Therefore the curve $\mathscr{C}$ exists and is parameterized by $x_{f}$. It can be determined by continuation, $x_{f} \mapsto x_{c}$ being solution of the Cauchy problem

$$
\frac{d x_{c}}{d x_{f}}=-\frac{\partial_{x_{f}} \phi^{\dagger}\left(x_{f}, x_{c}\right)}{\partial_{x} \phi^{\dagger}\left(x_{f}, x_{c}\right)}, \quad x_{c}\left(\bar{x}_{f}\right)=\bar{x}
$$

for $x_{f} \geq \bar{x}_{f}$. Computation gives the following expression

$$
\frac{d x_{c}}{d x_{f}}=\frac{A_{1} x_{c}+B_{1}}{A_{2} x_{c}-A_{1} x_{f}+B_{2}}
$$

with the parameters

$$
\begin{aligned}
& A_{1}=-c_{p}\left(a_{p} Q_{r}+a_{r} Q_{p}\right), B_{1}=b_{p} c_{p} Q_{r} \\
& A_{2}=2\left(a_{p} c_{r}-a_{r} c_{p}\right) Q_{p}, B_{2}=a_{p}\left(d_{r} Q_{p}+d_{p} Q_{r}\right)-b_{p} c_{r} Q_{p}
\end{aligned}
$$

One can easily check that the solution is given by

$$
\begin{aligned}
& x_{c}=\bar{x}+\frac{A_{1} x_{f}-B_{2}-A_{2} \bar{x}}{A_{2}}+ \\
& \frac{\sqrt{\left(A_{1} x_{f}-B_{2}-A_{2} \bar{x}\right)^{2}+2 A_{2}\left(A_{1} \bar{x}-B_{1}\right)\left(x_{f}-\bar{x}_{f}\right)}}{A_{2}}
\end{aligned}
$$

The curve $\mathscr{C}$ can be then determined by expressions (29) and (30) for $x_{f} \in\left[\bar{x}_{f}, x_{\max }\right)$, accordingly to Lemma 5.1.

\section{OPTIMAL SYNTHESIS}

We assume that there exists a single candidate singular arc, which seems to be realistic situations as illustrated in Section VII. Indeed, the absence of singular arc appears to be degenerate cases, with no trade-off between production and regeneration. Having several singular arcs imposes strong conditions on the parameters, which seem unrealistic.

Assumption 6.1: There exists an unique $\bar{x}$ among $\bar{x}_{\delta}$ and the roots of $N$ that belongs to $\left(0, x_{\max }\right)$ with $\bar{u} \in[-1,1]$, such that $\bar{\lambda}_{p}(\bar{x})>0$ and $\gamma_{\bar{x}}(\cdot)$ is increasing on $\left(0, x_{\max }\right)$.

In particular, this assumption is fulfilled when:

- $C$ given in (27) is positive,

- condition (24) is fulfilled and $\bar{x}$ given in (25) satisfies $\bar{x}<x_{\max }$ and $\bar{\lambda}_{p}(\bar{x})>0$, where $\bar{\lambda}_{p}(\cdot)$ is given in (19),

- $\bar{u}$ defined in (20) belongs to $(0,1)$,

- $\gamma_{\bar{x}}(\cdot)$ defined in $(26)$ is increasing on $\left(0, x_{\max }\right)$.

Proposition 6.1: Under Assumption 6.1, define the following partition of the domain $\mathscr{D}$ :

$$
\begin{aligned}
& \mathscr{S}=\{\bar{x}\} \times[0, \bar{p}](\text { where } \bar{p} \text { is given by }(28)) \\
& \mathscr{D}_{-}=\left\{(x, p) \in \mathscr{D}, x \leq x_{c}\left(x_{f}\right), x_{f} \in\left(\bar{x}_{f}, x_{\text {max }}\right]\right\} \\
& \mathscr{D}_{+}=\mathscr{D} \backslash(\mathscr{D} \cup \mathscr{S})
\end{aligned}
$$

Then, the following feedback control is optimal.

$$
u(x, p)=\left\{\begin{aligned}
+1, & (x, p) \in \mathscr{D}_{+} \\
\bar{u}, & (x, p) \in \mathscr{S}^{(} \text {where } \bar{u} \text { is given by (20)) } \\
-1, & (x, p) \in \mathscr{D}_{-}
\end{aligned}\right.
$$

Proof: At $(x, p)$ where $\phi=0, \lambda_{p}=\bar{\lambda}_{p}(x)$ given in (19) and has to be positive (Lemma 3.1). $\psi(x, p)$ is given by $\Gamma(x)$ defined in (23). As we have assumed that $N$ has only one root with positive $\bar{\lambda}_{p}$, we deduce that $\psi$ is not null when $x \neq \bar{x}$. The sign of $\Gamma$ for $x \neq \bar{x}$ has to be the same than $\gamma_{\bar{x}}$ (otherwise by continuity another root would exist), that is negative for $x<\bar{x}$ and positive for $x>\bar{x}$. From (22), the following properties are then satisfied.

- $\phi=0$ with $x<\bar{x} \Rightarrow \dot{\phi}<0$. $\phi$ can change its sign only when decreasing. Only switch from $u=1$ to $u=-1$ can be optimal in the sub-domain $\{x<\bar{x}\}$.

- $\phi=0$ with $x>\bar{x} \Rightarrow \dot{\phi}>0$. $\phi$ can change its sign only when increasing. Only a switch from $u=-1$ to $u=1$ can be optimal in the sub-domain $\{x>\bar{x}\}$.

Consider an initial condition with $x_{0}<\bar{x}$. The control $u=-1$ makes $x(\cdot)$ decreasing, remaining in $\{x<\bar{x}\}$ and then cannot switch to reach the target with $u=+1$, which contradicts its optimality (Lemma 3.1). We deduce that $u=+1$ is optimal at any $x<\bar{x}$. If the initial condition belongs to $\mathscr{D}_{+}$with $x_{0} \geq \bar{x}$, none of the solutions with $u=+1$ or $u=-1$ reaches a locus of commutation $\mathscr{S}$ or $\mathscr{C}$. Therefore, $u=+1$ is optimal until $t_{f}$. So, $u=+1$ is optimal when the state belongs to $\mathscr{D}_{+}$.

Consider now an initial condition in $\mathscr{D}_{-}$. The optimal control has to switch. Otherwise, it will be equal to +1 and as $x(\cdot)$ with $u=+1$ is increasing, it will have to cross the switching curve $\mathscr{C}$. So $u=-1$ is optimal until the trajectory reaches $\mathscr{C}$ (and then enters the sub-domain $\mathscr{D}_{-}$) or reaches $\mathscr{S} \cdot u=-1$ 
is thus optimal when the state belongs to $\mathscr{D}_{-}$.

Finally, when an optimal trajectory reaches $\mathscr{S}$ from below or from above, it has to stay on $\mathscr{S}$ with the control $\bar{u}$ until it exits $\mathscr{S}$. If not, it leaves $\mathscr{S}$ either with $u=-1$ and enters the sub-domain $\mathscr{D}_{+}$(but we have proved that $u=+1$ is optimal on this domain), either with $u=1$ and enters the sub-domain $\mathscr{D}_{-}$(and $u=-$ is optimal on this domain).

\section{APPLICATION}

We consider a filtration system and give the complete synthesis with the construction of the switching curve given in Section V. It is usually assumed that the transmembrane pressure (TMP) $\tau_{p}$ and $\tau_{r}$ during filtration and backwash are positive functions obtained Darcy's law, as:

$$
\tau_{p}(x)=\frac{Q_{p}}{A} \mu R_{t o t}(x), \quad \tau_{r}(x)=\frac{Q_{r}}{A} \mu R_{t o t}(x)
$$

with $Q_{p}$ and $Q_{r}$ are the filtration and the backwash flow rates, respectively, and $A$ the membrane area. Practically the backwash flow rate is considered higher or equal to the filtration rate. Then, one can write $Q_{r}=c Q_{p}$ with $c>1$. Parameters values are given in Table VII.

\begin{tabular}{|l|l|l|l|l|l|l|l|l|}
\hline$a_{p}$ & $b_{p}$ & $c_{p}$ & $d_{p}$ & $c_{r}$ & $d_{r}$ & $Q_{p}$ & $Q_{r}$ & $p^{*}$ \\
\hline 0 & 1 & 0.16 & 3 & 0.64 & 12 & 1 & 2 & 50 \\
\hline
\end{tabular}

Assumption 2.1 is clearly fulfilled on $(0,+\infty)$. The discriminant $\Delta(x)$ of system (18) is given by

$$
\Delta(x)=0.5 x-2
$$

and null for $\bar{x}_{\Delta}=4$. A straightforward computation gives $\bar{\lambda}_{p}\left(x_{\Delta}\right)=-1.75<0$. From Assumption 6.1 we conclude that $x_{\Delta}$ can not be a candidate singular arc. Moreover, one has:

$$
N(x)=-0.04 x^{2}+0.32 x+10.2
$$

which can have two changes of sign at $\bar{x}_{-}=-12.5$ and $\bar{x}_{+}=20.5 . \bar{x}_{+}=20.5$ is the unique singular arc in $\mathscr{D}$ with $\bar{u}=-0.82 \in[-1,1]$ such that $\bar{\lambda}_{p}(\bar{x})=10.75>0 . \gamma_{\bar{x}}(\cdot)$ is increasing on $(0,+\infty)$ according to its expression

$$
\gamma_{\bar{x}}(x)=\frac{0.04 x^{2}+0.16 x+20}{x+2}
$$

The singular arc is left for $\bar{p}=22$ and $\bar{x}_{f}=48.44$. The criterion value to obtain the production $p *$ in minimum time is then equal to $E_{T}=420 \mathrm{Wh}$. The curve $\mathscr{C}$ is given by

$$
x_{c}=0.5 x_{f}-3.75-6.25 \sqrt{0.0064 x_{f}^{2}-0.2 x f-5.4}
$$

Figure 1 illustrates the optimal feedback synthesis.

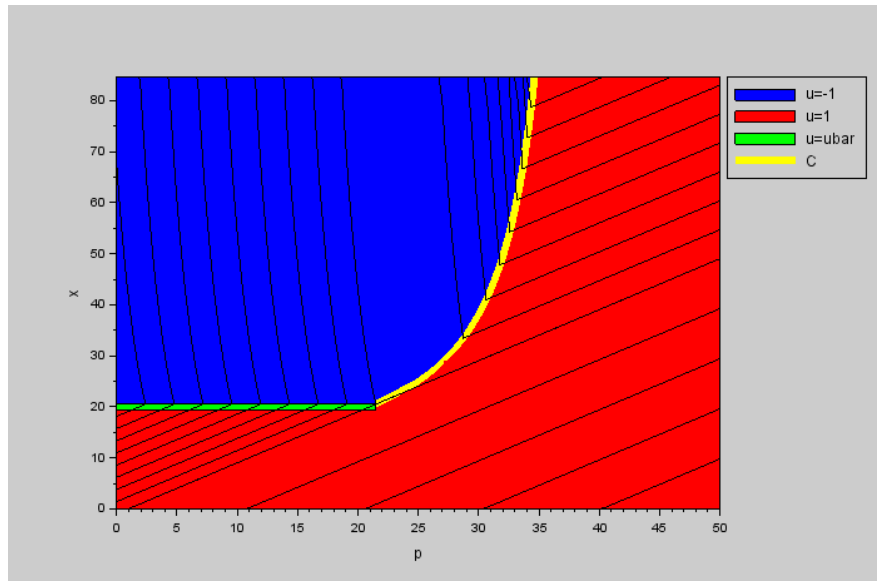

Fig. 1. Optimal synthesis for the considered parameters in the $(p, x)$ plane. The singular arc is in green and the switching curve in yellow.

\section{CONCLUSION}

We have proposed a methodology to determine an optimal feedback synthesis. This synthesis looks similar to the one of filtration problems with fixed terminal time [5], but the determination of the singular arc is here much more complex, requiring the consideration of the auxiliary functions $\Delta, \Gamma, \bar{\lambda}_{p}$ and $\gamma_{\bar{x}}$. For linear dynamics, this leads to explicit polynomials whose coefficients can be determined analytically from the parameters of the problem. A future work will investigate extensions of the results to more general classes of dynamics.

\section{ACKNowledgments}

This work was supported by the Islamic Bank of Development which granted the $\mathrm{PhD}$ of $\mathrm{F}$. Aichouche and the French National Research Agency within \#DigitAg program, ANR-16-CONV-0004 who provided the postdoc grant of N. Kalboussi. The authors thank also the Euro-Mediterranean network TREASURE (www.inra.fr/treasure.)

\section{REFERENCES}

[1] T. Sparks and G. Chase, Filters and Filtration Handbook, ButterworthHeinemann, 2015.

[2] A. Robles, M. V. Ruano, A. Seco, J.-P. Steyer, J. Harmand, A. Charfi, J. Kim, M. Heran, G. Lesage, D. J. Batstone, J. Ferrer A review on anaerobic membrane bioreactors (AnMBRs) focused on modelling and control aspects. Bioresource Technology, 270, pp.612-626, 2018.

[3] Y. Lan, K. Groenen-Serrano, C. Coetsier, and C. Causserand, Fouling control using critical, threshold and limiting fluxes concepts for crossflow NF of a complex matrix: Membrane BioReactor effluent, J. Memb. Sci., vol. 524, no. May 2016, pp. 288-298, 2017.

[4] J. Harmand, C. Lobry, A. Rapaport and T. Sari, Optimal Control in Bioprocesses: Pontryagin's Maximum Principle in Practice, WileyISTE, 2019.

[5] N. Kalboussi, A. Rapaport, T. Bayen, N. Ben Amar, F. Ellouze and J. Harmand, Optimal control of membrane filtration systems, IEEE Transactions on Automatic Control. Vol. 64 (5), 2019.

[6] M. Robles, K. Lee, and J.-S. Kim, Optimal Control based Charging/Discharging Strategy of Dual Battery Energy Storage System for Wind Power Dispatch, Proceedings of the SICE Annual Conference, Sept. 19-22, 2017, Kanazawa, Japan. 